\title{
Does a True Fatigue Limit Exist for Continuous Fiber-Reinforced Ceramic Matrix Composites?
}

\author{
Bent F. Sørensen
}

Materials Research Department, Risø National Laboratory, DK-4000 Roskilde, Denmark

\author{
John W. Holmes ${ }^{\dagger}$ and Eddy L. Vanswijgenhoven ${ }^{\dagger}$ \\ Ceramic Composites Research Laboratory, Department of Mechanical Engineering and Applied Mechanics, \\ University of Michigan, Ann Arbor, Michigan 48109-2125
}

\begin{abstract}
The high-cycle high-frequency fatigue behavior of a Nicalonfiber-reinforced calcium aluminosilicate ceramic composite was investigated. A key goal of the room-temperature fatigue experiments was to determine if a true fatigue limit or endurance limit existed for this ceramic matrix composite. Although no fatigue failures occurred beyond $10^{7}$ cycles, the stress-strain hysteresis modulus and frictional heating continued to change up to $10^{8}$ cycles, at which point the $200 \mathrm{~Hz}$ experiments were terminated. This suggests that fatigue damage continued to evolve and that a true fatigue limit may not exist in ceramic matrix composites that have undergone interfacial frictional sliding.
\end{abstract}

\section{Introduction}

$\mathrm{C}$ ERAMICS reinforced with continuous fibers can possess damage tolerance, because the first mode of microstructural damage (multiple matrix cracking in association with fiber/matrix debonding and sliding) does not lead to fracture. Indeed, fiber-reinforced ceramic matrix composites (CMCs) may possess completely notch-insensitive behavior. ${ }^{1}$ This class of materials is expected to be used in load-carrying structures at high temperatures; potential applications involve changes in load as a function of time. ${ }^{2,3}$ Although debonding and sliding along fiber/matrix interfaces (or between interphase layers) in a CMC is a necessity for damage-tolerant behavior, it may be detrimental under cyclic loading; repeated forward and backward sliding may result in wear damage to the interface/ interphase regions of the composite. Unless the wear process ceases, the CMCs may experience fatigue failure caused by cyclic wear damage. The occurrence of this damage process is a fundamental interface problem that is highly relevant for the high-temperature behavior of future CMC systems, which are likely to possess oxidation-resistant interphases. From a practical viewpoint, the question is whether CMCs possess a finite fatigue life (in analogy to aluminum alloys) or whether an endurance fatigue limit exists for an infinite number of load cycles (in analogy to that found for low-carbon steels). Despite

R. J. Kerans-contributing editor

Manuscript No. 189761. Received July 12, 2000; approved July 23, 2001.
Supported by the National Science Foundation (Grant No. DMR-9257557) and the Supported by the National Science Foundation (Grant No. DMR-9257.
Air Force Office of Scientific Research (Grant No. F49620-95-1-0206).

Partially supported by Ris $\emptyset$ Engineering Science Center for Structural Characterization and Modeling of Materials.

${ }^{\star}$ Member, American Ceramic Society.

Present address: Department of Engineering Science and Mechanics, The Pennsylvania State University, University Park, Pennsylvania 16802. a number of experimental studies on the fatigue behavior of CMCs (see, e.g., an overview of the fatigue of CMCs) ${ }^{3-5}$ the question of whether fatigue damage eventually stabilizes remains unanswered. If an endurance fatigue limit does exist for CMCs, then research should be aimed at identifying the critical conditions that separate the occurrence of fatigue failure and infinite fatigue life. If a true fatigue limit does not exist, then models should be focused toward predicting fatigue life through rate laws that describe fatigue damage.

In many studies of the fatigue behavior of CMCs, a fatigue limit (i.e., the maximum allowable stress that a composite can withstand without the occurrence of fatigue failure) is defined at a specific number of cycles (typically $10^{5}$ or $10^{6}$ cycles), regardless of the fact that the surviving specimens develop distributed damage. Attempts have been made to correlate the fatigue limit, $\sigma_{\mathrm{fl}}$, to characteristic features of the monotonic stress-strain curve. $^{6-9}$ This is difficult, however, because the evolution of damage under monotonic tension and cyclic loading may depend on loading velocity. ${ }^{10,11}$ Moreover, the damage mechanisms that control strength degradation under cyclic loading may be different from the damage mechanisms that operate during monotonic tension. Indeed, a literature survey shows that, in general, the onset of nonlinearity in a monotonic stress-strain curve and $\sigma_{\mathrm{fl}}$ do not coincide. ${ }^{3}$ Tests performed at room temperature show that, for CMCs with weakly bonded fiber/matrix interfaces, fatigue life decreases with decreasing stress ratio $^{12,13}$ and with increasing loading frequency. ${ }^{11,14}$

During cyclic loading, the evolution of fatigue damage results in measurable changes in properties. For conventional CMCs with weakly bonded interfaces, multiple matrix cracking in connection with interfacial debonding and sliding results in decreasing stiffness, permanent strain, and hysteresis. ${ }^{7,9,15-17}$ The latter can result in a significant temperature rise due to frictional heating. ${ }^{11,14,18,19}$

Several analytical models have been developed to explain the above-mentioned phenomena. ${ }^{8,16,20}$ In most of these models, the interface has been modeled as purely frictional with a constant interfacial shear stress, $\tau$. Frictional heating by interfacial sliding in a CMC has been modeled by Cho et al. ${ }^{21}$ Their model has been used to calculate how $\tau$ changes during the fatigue of a unidirectional $\mathrm{SiC}_{\mathrm{f}} / \mathrm{CAS}$ II composite. ${ }^{19} \tau$ decreases rapidly, from $>20$ to $\sim 5 \mathrm{MPa}$, in the early stages of fatigue. This decrease is attributed to abrasive wear along the fiber/ matrix interface as the fiber and matrix repeatedly slide against each other. ${ }^{19,22,23}$ After this initial decrease, $\tau$ remains approximately constant with continued cycling. Models used to predict the strength of a fiber-reinforced ceramic suggest that composite strength decreases with decreasing fiber strength and decreasing $\tau .^{24-27}$ Rouby and Reynaud ${ }^{27}$ have proposed that fatigue failures are caused by a decrease in $\tau$, in connection with 
sliding wear along the fiber/matrix interface. However, a decrease in $\tau$, acting alone, does not adequately explain the fatigue failures of CMCs. For instance, as mentioned above, early work on the fatigue of $\mathrm{SiC}_{\mathrm{f}} / \mathrm{CAS}$ II showed that $\tau$ decreases within the first 25000 fatigue cycles from $>20 \mathrm{MPa}$ to a constant level of 5-6 $\mathrm{MPa} .{ }^{19}$ Fatigue failure occurs beyond $3 \times 10^{6}$ cycles, even though $\tau$ and matrix crack spacing change only slightly after 25000 cycles. Another possible fatigue damage mechanism is cyclic-slip-induced wear damage to the surface of the fibers. ${ }^{28,29}$ In CMCs with woven fiber architectures, damage may occur at points where fiber bundles cross over each other. ${ }^{30}$ Fatigue damage may also interact with time-driven phenomena, such as the oxidation or stress corrosion of fibers. ${ }^{31}$

There have been, as far as we know, no systematic studies undertaken to determine how fatigue damage evolves for fatigue loading significantly exceeding $10^{6}$ cycles. Therefore, in this study, fatigue experiments are performed for up to $10^{8}$ cycles at loading frequencies of 200 and $500 \mathrm{~Hz}$. Key damage indicators are measured and related to the damage state of the composite. These damage indicators are the average matrix crack spacing, $s$; the hysteresis modulus, $\bar{E}$ (defined from the maximum and minimum stress-strain data points during a load cycle); the permanent strain, $\varepsilon^{*}$; and the temperature rise, $\Delta T$ (see Fig. 1). These damage indicators can be related to microstructural parameters through micromechanical models. ${ }^{16,20,21}$ Note that these damage indicators are measured over a specified volume. If damage evolves nonuniformly within the specimen, other parameters sensitive to local variations should be used (e.g., local crack opening). The key questions we seek to answer are

(1) Can an endurance fatigue limit be identified for the fatigue of fiber-reinforced CMCs where frictional slip occurs?

(2) What microstructural damage can occur without causing fatigue failures, and what mode of microstructural damage results in fatigue failure?

\section{Experimental Procedure}

A 16-ply unidirectional Nicalon ${ }^{\mathrm{TM}}$ (Nippon Carbon Co., Tokyo, Japan) silicon-carbide-fiber-reinforced calcium aluminosilicate, with a fiber volume fraction of 0.31 , was used in the investigation. For brevity, we denote the composite as $\mathrm{SiC}_{\mathrm{f}} / \mathrm{CAS}$ II (Corning, Inc., NY). The $\mathrm{SiC}_{\mathrm{f}} / \mathrm{CAS} \mathrm{II}$ composite was formed into rectangular billets by hot-pressing. Edge-loaded tensile specimens with a $33 \mathrm{~mm}$ gauge length were cut from the billets by diamond tooling so that the tensile direction was parallel to the fiber direction (Fig. 2). To allow observation of matrix cracking, one of the minor faces of each specimen was polished. Polishing was performed using a $38 \mathrm{~mm}$ mandrel rotating at $1500 \mathrm{rpm}$. The following polishing procedure was used: (1) 600-grit SiC paper for $5 \mathrm{~min}$, (2) $45 \mu \mathrm{m}$ diamond paste for $5 \mathrm{~min}$ (nylon cloth), (3) $6 \mu \mathrm{m}$ diamond paste for $5 \mathrm{~min}$ (nylon cloth), and (4) $1.0 \mu \mathrm{m}$ diamond paste for $10 \mathrm{~min}$ (nylon cloth).

Monotonic tension tests were conducted at a constant loading rate of $100 \mathrm{MPa}^{-1}$ on a MTS servo-hydraulic load frame (Model 810, MTS Systems Corp., Minneapolis, MN). A rapid loading rate was chosen for two reasons. First, a rapid loading rate minimizes the effect of stress corrosion on matrix cracking. ${ }^{19,32}$ Second, the interfacial sliding velocity would be high. (The interfacial shear stress, operating during fiber/matrix sliding, has been shown to be velocity-dependent. $\left.{ }^{10,33}\right)^{\ddagger}$ Axial strain data were measured using an extensometer with a $33 \mathrm{~mm}$ gauge length.

Tension-tension fatigue experiments were conducted on a servohydraulic load frame (Model 331, MTS Systems) equipped with low-mass edge-loaded grips (Composite Testing and Analysis, State College, PA). ${ }^{34}$ Before the experiments, specimen alignment was checked by built-in piezoelectric load cells in the upper grip. The fatigue experiments were conducted under load control using a sinusoidal wave form with a frequency of 200 or $500 \mathrm{~Hz}{ }^{\S}$ To prevent overshooting of the desired load in the first few cycles, the load span was increased (linearly with time) from zero to the maximum stress value, $\sigma_{\max }$, within $0.8 \mathrm{~s}$. Fatigue experiments were conducted using different maximum stress levels. For all experiments, the lower stress level, $\sigma_{\min }$, was $10 \pm 5 \mathrm{MPa}$.

\footnotetext{
${ }^{*}$ Note that interface velocity is still not as high as that encountered by the fiber/matrix interface during typical fatigue test.

${ }^{\S}$ Simple estimate (see Appendix) was made of the magnitude of dynamic stresses due to high loading frequency. Maximum dynamic stress was $<1 \mathrm{MPa}$ and was neglected in remainder of paper.
}

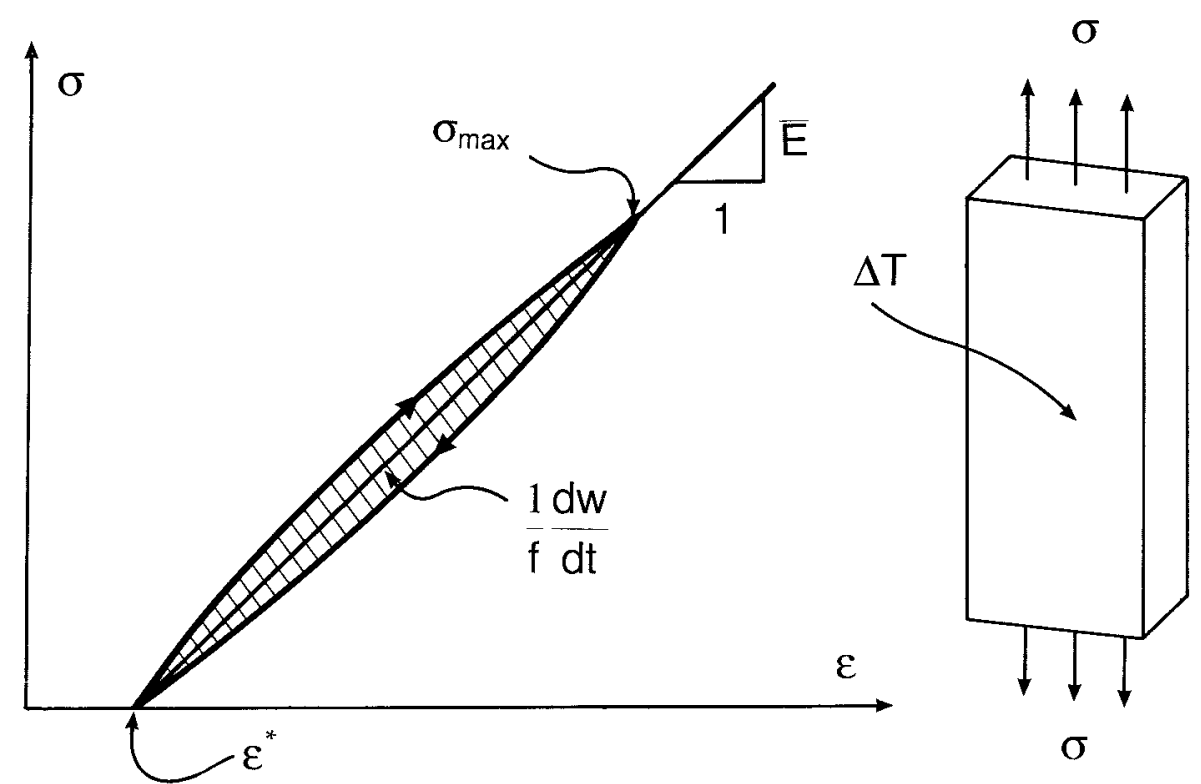

(a)

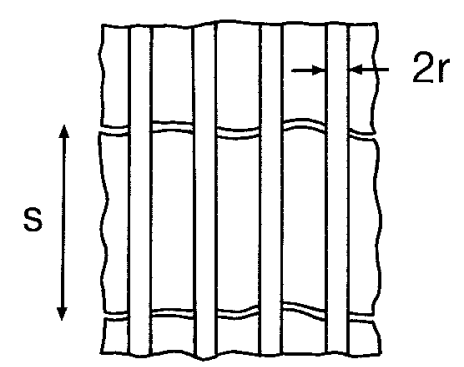

(b)

Fig. 1. Damage indicators for unidirectional CMCs: (a) cyclic stress-strain curve defines the hysteresis modulus, $\bar{E}$, and permanent strain, $\varepsilon^{*}$. (b) At surface of specimen, temperature rise, $\Delta T$, and matrix cracks (shown with regular matrix crack spacing, $s$ ) are primary damage indicators. 


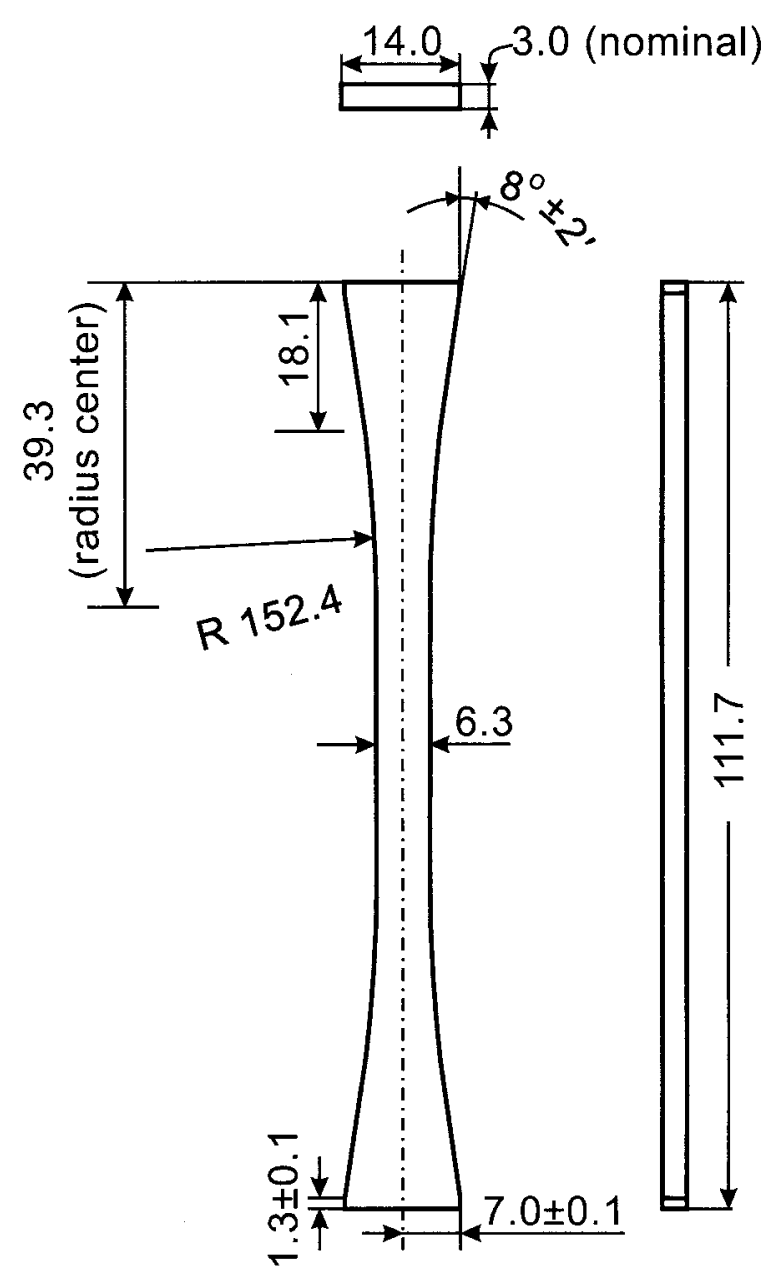

Fig. 2. Edge-loaded tension-tension fatigue specimens used in this study. All dimensions are in $\mathrm{mm}$.

All fatigue tests were performed inside a $0.1 \mathrm{~m}^{3}$ water-cooled chamber (Composite Testing and Analysis). ${ }^{34}$ The temperature of the chamber walls and edge-loaded grips was kept constant at $22.0 \pm 0.1^{\circ} \mathrm{C}$. The temperature of the specimen surface was measured by infrared pyrometers (Model 5402 for temperatures $<70^{\circ} \mathrm{C}$, Model 5430 for temperatures $>70^{\circ} \mathrm{C}$, Everest Interscience, Inc., Fullerton, CA), focused at a $5 \mathrm{~mm}$ spot size within the specimen gauge section. The pyrometers had a resolution of $0.1^{\circ}$ and $0.5^{\circ} \mathrm{C}$, respectively, and a response time of $300 \mathrm{~ms}$. Temperature data were collected with a 16-bit resolution data acquisition system (Model ACM2-16, Strawberry Tree Computers, Sunnyvale, CA). To achieve stable conditions within the test chamber, the temperature was allowed to stabilize for at least $2 \mathrm{~h}$ before the start of the fatigue test. Axial strain data were measured by an extensometer (Model 632.27B-20, MTS Systems) with a $33 \mathrm{~mm}$ gauge length. The extensometer was mounted along a specimen edge by $\mathrm{O}$ rings, and the knife edges were secured to the specimen by superglue. Signals from the load cell and extensometer were gathered by an additional high-speed 16-bit resolution data acquisition system (with 16-bit, $50 \mathrm{kHz}$ daughter boards; Model MBC-625, GW Instruments, Sommerville, MA). Stress-strain hysteresis loop data were recorded at regular intervals. From this data, the $\bar{E}$ (averaged over one cycle, see Fig. 1(a)) was calculated as a function of the number of load cycles.

After the fatigue tests, which were performed until failure or $10^{8}$ cycles (run-out), the number of cracks along a line parallel to the fiber direction were counted using an optical microscope. In most cases, more than 300 matrix cracks were counted for each specimen. Specimens surviving $10^{8}$ cycles were loaded in monotonic tension (loading rate $100 \mathrm{MPa} \cdot \mathrm{s}^{-1}$ ) to assess their residual strength. Fracture surfaces were inspected by optical and scanning electron microscopy.

\section{Experimental Results}

\section{(1) Monotonic Stress-Strain Behavior}

For many CMC systems, the stress-strain curve during monotonic tensile loading can be divided into four characteristic stages, reflecting the underlying damage evolution. $3,10,35,36$ At low applied stress, no damage evolves, and the behavior is truly linearly elastic (Stage I). At higher stresses, matrix cracks evolve along with fiber/matrix debonding and sliding (Stage II). Matrix cracking usually saturates, and a characteristic damage state consisting of fairly regularly spaced fiber-bridged matrix cracks forms. Beyond saturation (Stage III), the stress-strain curve usually regains linearity. The majority of the stress is carried by the fibers, which slide along fiber/matrix interfaces. Distributed fiber failures (Stage IV) may occur before deformation localization (i.e., when self-sustaining loss of strength occurs in a narrow band, leading to the formation of the fracture surface and specimen separation).

The room-temperature tensile stress-strain curve of $\mathrm{SiC}_{\mathrm{f}} /$ CAS II (Fig. 3) exhibits distinct nonlinearity due to distributed damage. The nonlinearity within Stage II is quantified by the stress level, $\sigma_{0.02}$, defined as the stress level where measured strain differs by $0.02 \%$ from linear elasticity. ${ }^{6}$ It is well known, e.g., from studies using acoustic emission, that the formation of the first matrix cracks do not cause measurable changes in specimen stiffness. ${ }^{35}$ Therefore, $\sigma_{0.02}$ does not represent the onset of matrix cracking. However, $\sigma_{0.02}$ is a well-defined experimental measure of the nonlinearity of the stress-strain curve. Moreover, $\sigma_{0.02}$ can be related to microstructural parameters. ${ }^{10}$ For a loading rate of $100 \mathrm{MPa} \cdot \mathrm{s}^{-1}, \sigma_{0.02}$ is measured to be $\sim 380 \mathrm{MPa}$. This is slightly higher than that found for $\mathrm{SiC}_{\mathrm{f}} / \mathrm{CAS}$ II in other studies for this loading rate. ${ }^{19}$ The stress-strain curve of our specimens does not possess the very distinct second linear region (Stage III) often found for $\mathrm{SiC}_{\mathrm{f}} /$ CAS II. ${ }^{16,19,35,36}$ These differences are attributed to differences between material billets used in the investigations.

The average crack spacing was $\sim 0.19 \mathrm{~mm}$. This is slightly higher $(\sim 20 \%)$ than that found in previous experiments conducted at similar loading rates. ${ }^{10}$ The average fiber pull-out length is measured to be $\sim 100 \mu \mathrm{m},{ }^{10}$ reasonably close to results from earlier investigations. ${ }^{36}$

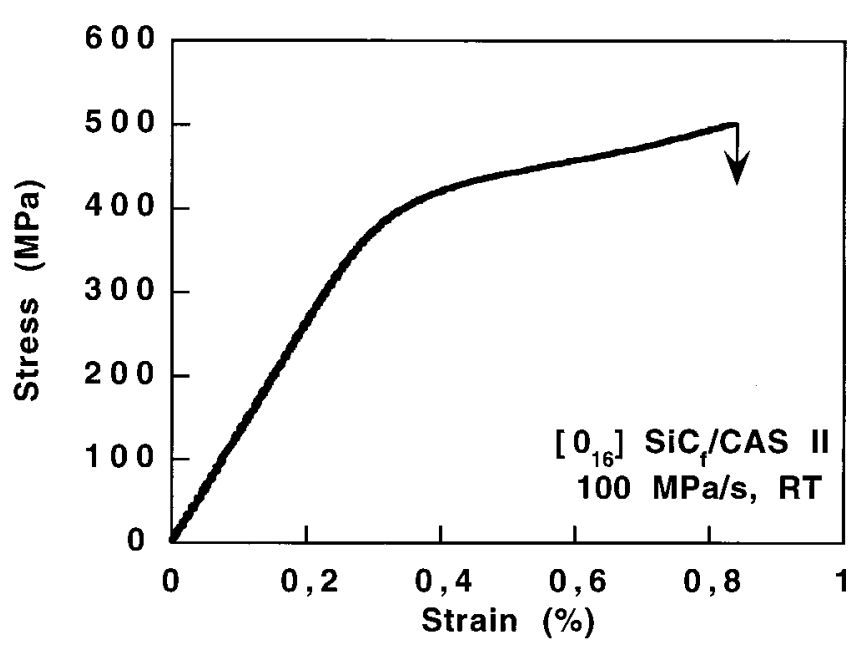

Fig. 3. Typical monotonic stress-strain curve for $\left[0_{16}\right]-$ Nicalon $^{\mathrm{TM}} \mathrm{SiC}_{\mathrm{f}} /$ CAS II composite used in this study. (To obtain well-defined behavior, tensile tests were performed at a loading rate of $100 \mathrm{MPa} \cdot \mathrm{s}^{-1}$.) The $0.02 \%$ proportional stress, $\sigma_{0.02}$, was $380 \mathrm{MPa}$. Nonlinear behavior is attributed to formation of multiple matrix cracks, fiber/matrix debonding, and interface slip. 


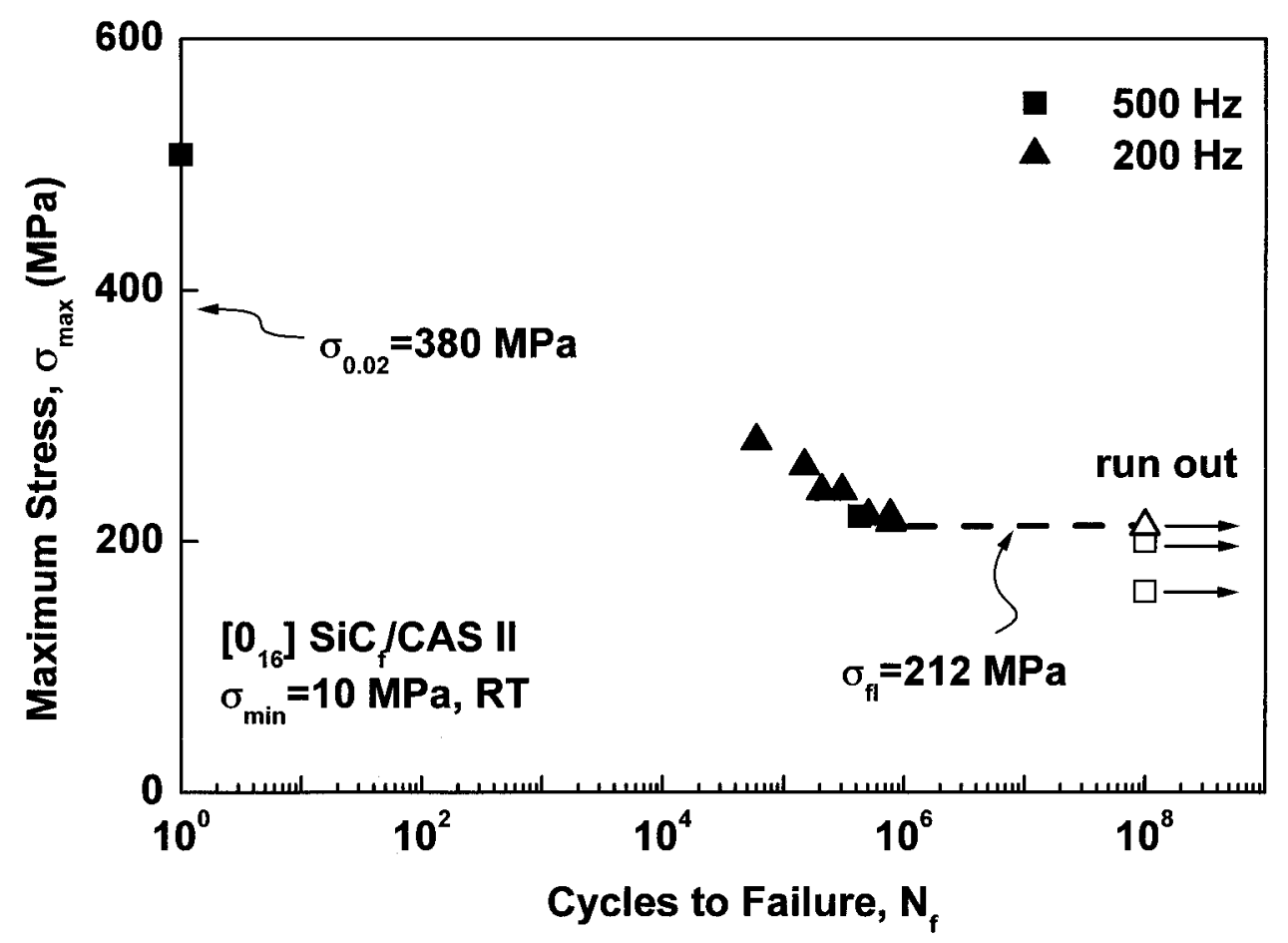

Fig. 4. $S-N$ curve for unidirectional $\mathrm{SiC}_{\mathrm{f}} / \mathrm{CAS}$ II composites. There is sharp demarcation in stress level between specimens that fail in fatigue and specimens that survive $10^{8}$ cycles. All failures occurred at stress levels below the $\sigma_{0.02}$ value determined by monotonic tensile tests. Run-out fatigue limit at $10^{8}$ cycles is $212 \mathrm{MPa}$. Note importance of properly defining fatigue limit. Had fatigue limit been defined at $10^{5}$ cycles, one would be tempted to incorrectly characterize material as having infinite life for stresses below $\sim 280 \mathrm{MPa}$.

\section{(2) Fatigue Behavior}

The fatigue life diagram (or $S-N$ curve) is shown in Fig. 4. There are several features of the curve. First, as expected, a higher $\sigma_{\max }$ results in a shorter fatigue life. Second, there is a rather narrow band of stress that separates specimens that failed during cycling and specimens that survived $10^{8}$ cycles. For $\sigma_{\min }=10$ $\mathrm{MPa}$, the run-out fatigue limit, $\sigma_{\mathrm{fl}}$, is identified as $212 \mathrm{MPa}$ for $10^{8}$ cycles and a loading frequency of $200 \mathrm{~Hz}$. Third, from Fig. 4, it appears that if fatigue failure occurs, it takes place within the first few million load cycles.

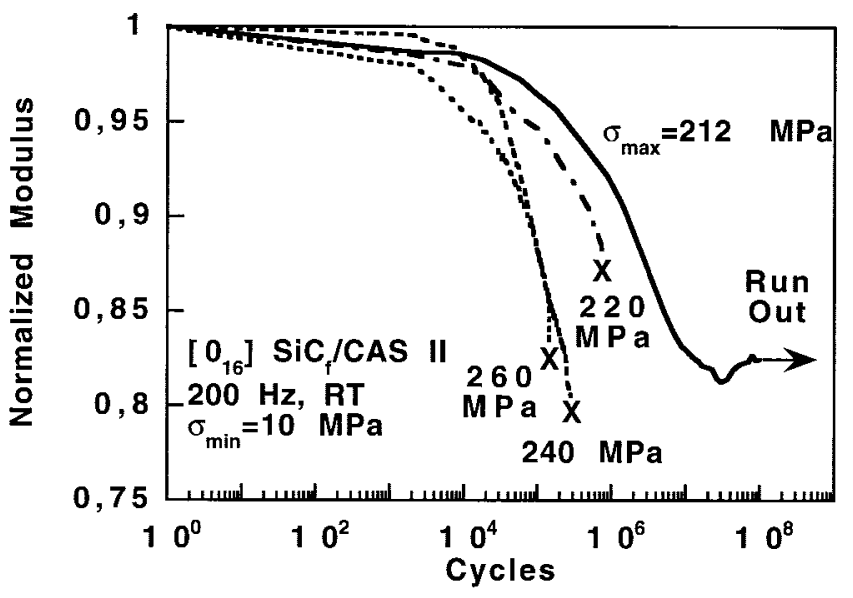

Fig. 5. Hysteresis modulus, $\bar{E}$ (normalized with initial modulus $E_{c}=131$ $\mathrm{GPa}$ ) of two specimens cycled under different $\sigma_{\max }$. Specimen loaded to highest $\sigma_{\max }$ shows faster modulus decrease, indicating that formation of matrix cracks occurs more rapidly at higher maximum stress. However, modulus decrease measured before fatigue failure at $\sigma_{\max }=220 \mathrm{MPa}$ is lower than modulus decrease for run-out specimen $\left(\sigma_{\max }=212 \mathrm{MPa}\right)$. Thus, changes in modulus are not accurate predictors of incipient fatigue failure.
The hysteresis modulus, $\bar{E}$, calculated from the stress-strain data is shown for two specimens in Fig. 5 as a function of the number of cycles. For a composite made of continuous fibers experiencing interfacial sliding, $\bar{E}$ depends on the stress range $\left(\Delta \sigma=\sigma_{\max }-\sigma_{\min }\right)$, as elaborated elsewhere. ${ }^{20,37}$ However, for two of the experiments shown in Fig. 5, the stress ranges are comparable $(\Delta \sigma=198$ and $211 \mathrm{MPa})$. For both specimens, the modulus decreased gradually with increasing cycles. The specimen experiencing the highest $\sigma_{\max }(220 \mathrm{MPa})$ failed within 10 cycles, ${ }^{6}$ whereas the other specimen $\left(\sigma_{\max }=212 \mathrm{MPa}\right)$ survived 10 cycles. ${ }^{8}$ The $\bar{E}$ of the surviving specimen decreased and stabilized; a minor recovery in the modulus occurred after $\sim 30 \times 10^{6}$ cycles. (Modulus recovery has previously been observed during the fatigue of other continuous fiber-reinforced ceramics. $)^{7,19,28-30,38}$ The rate at which the modulus decreased was greatest for the specimen fatigued at the highest $\sigma_{\max }$. However, one of the largest modulus decrease was found for the specimen that survived $10^{8}$ cycles. Thus, the magnitude of the modulus decrease in itself cannot be used as a predictor of whether fatigue failure will occur.

The temperature rise, $\Delta T$, of several specimens is shown in Fig. 6 as a function of the number of cycles. For the specimen subjected to $\sigma_{\max }=220 \mathrm{MPa}$, the temperature rose monotonically to failure. For the specimen run at $\sigma_{\max }=212 \mathrm{MPa}$, the temperature increased by $\sim 25^{\circ} \mathrm{C}$ (almost as high as the temperature rise measured at failure for the other specimen) and decreased thereafter. This specimen survived $10^{8}$ cycles. The changes in $\Delta T$ were attributed to changes in $\tau$ and the nature of the interface (e.g., accumulation of wear debris).

In general, for specimens that failed within the detection zone of the infrared pyrometer, a sharp temperature rise was measured during the last few thousand cycles before failure. Such a rapid temperature rise was the best predictor of fatigue failure. This rapid temperature rise is attributed to localized fiber failures, which are assumed to increase the effective fiber slip length within the localized region. The fact that such a temperature rise occurs just before fatigue failure suggests that, 


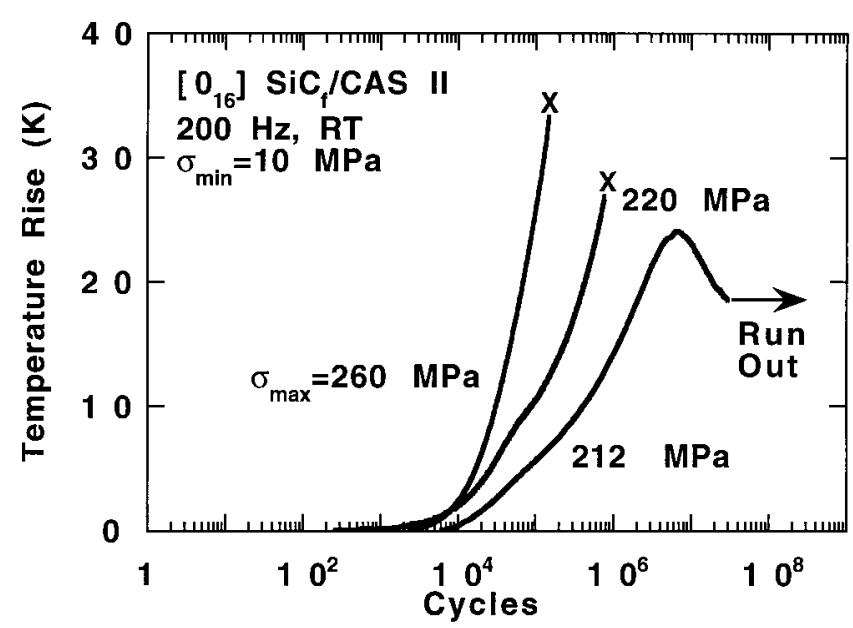

Fig. 6. Temperature rise, $\Delta T$, of three of the specimens described in Fig. 5. Specimens that do not fail during cycling show temperature rise curve that increases initially, stabilizes, and then decreases again, i.e., "bellshaped" curve.

during high-frequency fatigue at moderate stress levels, a significant number of fiber failures occur only near the very end of fatigue life. Identifying the mechanism responsible for triggering these fiber failures is the key to understanding and controlling fatigue failure.

The evolution of permanent strain, $\varepsilon^{*}$, as a function of the number of cycles, is shown in Fig. 7 for several specimens. In this figure, $\varepsilon^{*}$ is defined as the strain value at $\sigma_{\text {min }}$ (nominally $10 \mathrm{MPa}$ in the current paper). The difference in $\varepsilon^{*}$ extrapolated down to $\sigma_{\text {min }}=0 \mathrm{MPa}$ is $\sim 0.01 \%$. From Fig. 7 , it is seen that $\varepsilon^{*}$ increases monotonically during cycling. The primary cause for this appears to be the formation of an increasing number of matrix cracks. (Both experimental ${ }^{15,35}$ and model studies ${ }^{16,20}$ indicate that $\varepsilon^{*}$ increases with decreasing matrix crack spacing, s.)

The average residual tensile strength of specimens surviving $10^{8}$ cycles is $437 \pm 30 \mathrm{MPa}$. This is $\sim 85 \%$ of the tensile strength of the virgin material. For comparison, specimens cycled to $10^{5}$ cycles retain their strength completely. ${ }^{6,37,39}$ The fact that specimens cycled to $10^{8}$ cycles have lower strength suggests the existence of a separate high-cycle fatigue damage mechanism that operates during high-frequency fatigue at low stress levels.

\section{(3) Microstructural Characterization}

The fracture surface of the specimens loaded in monotonic tension (virgin and run-out specimens) displays considerable fiber pull-out across the entire cross section. This is typical of damage-tolerant CMCs. ${ }^{3,10,36}$ In contrast, the fracture surface of the specimens that fail during cycling has an interior core region where there is no fiber pull-out (Fig. 8), while the surrounding fracture surface displays fiber pull-out. The fact that all fibers in the central region fail in the same fracture plane indicates that the interface changes and that fiber sliding is hindered. Consequently, no global load sharing could have taken place between the fibers; the zone fractures like a brittle material. It is tempting to speculate that the embrittled zone is caused by internal heating (the bulk temperature inside the specimen is likely to exceed that of the surface, where heat is lost by radiation and convection). This is discussed in detail in another paper. ${ }^{39}$

Values for the matrix crack density of the fatigued specimens are given in Table I. For the specimens cycled at 160 and $200 \mathrm{MPa}$, matrix cracking was mainly restricted to the matrix-rich regions. For the other specimens (having been subjected to a higher maximum stress), matrix cracks were long and bridged by intact fibers. Generally, matrix crack density increased with $\sigma_{\max }$ and the number of cycles (the latter, perhaps owing to stress corrosion cracking). ${ }^{32}$ The specimen that survived $10^{8}$ cycles at $\sigma_{\max }=212$ MPa had the highest crack density, 5.7 cracks per mm. From Table $\mathrm{I}$, it is apparent that there is no correlation between the occurrence of fatigue failure and matrix crack density.

To examine internal damage, a specimen that had been cycled to failure was cut and polished along its axial mid-plane, so that the polished surface was parallel with a broad face. Matrix cracks were distributed uniformly inside the specimen and also inside the embrittled zone. The matrix crack density measured in the interior was similar to that measured at the

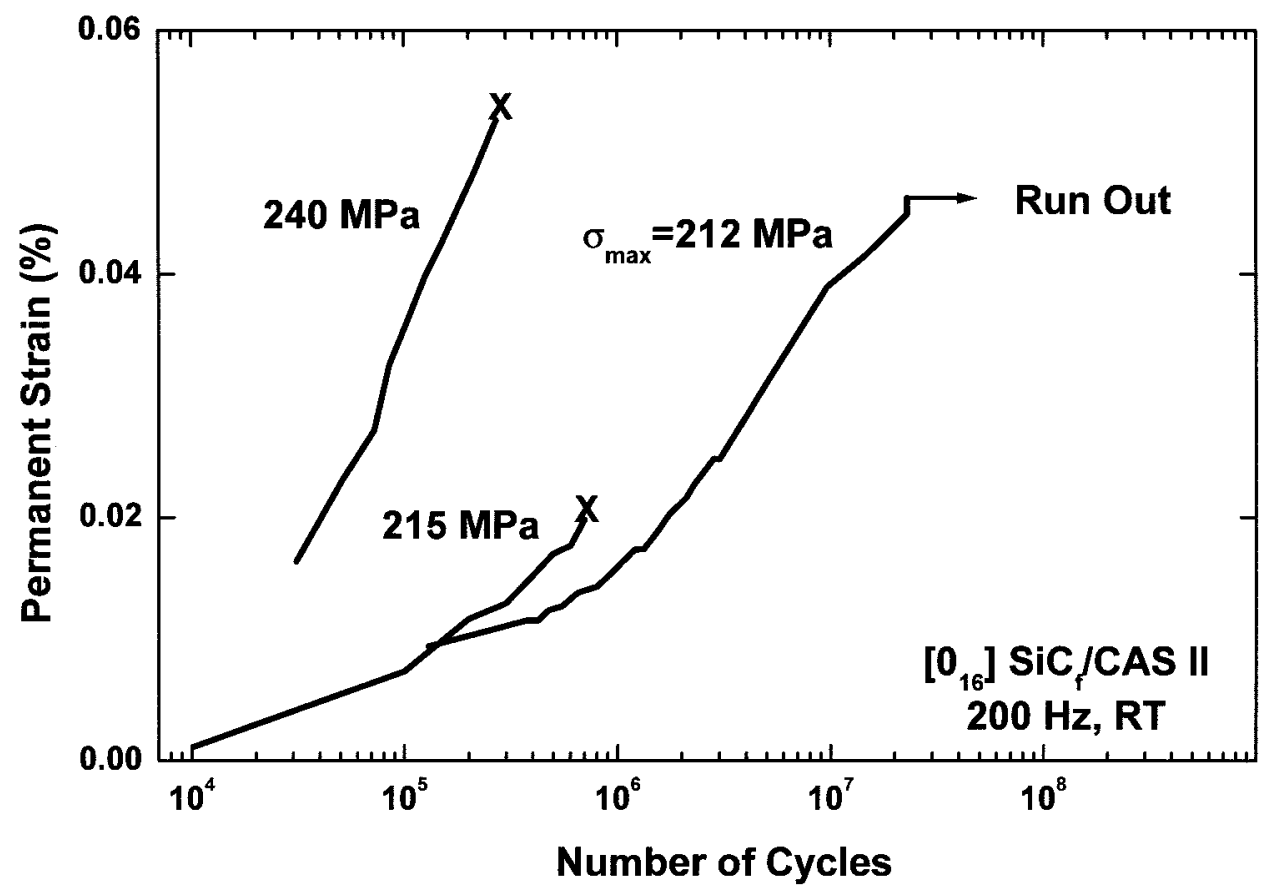

Fig. 7. Permanent strain, $\varepsilon^{*}$, as function of number of cycles for some specimens. 

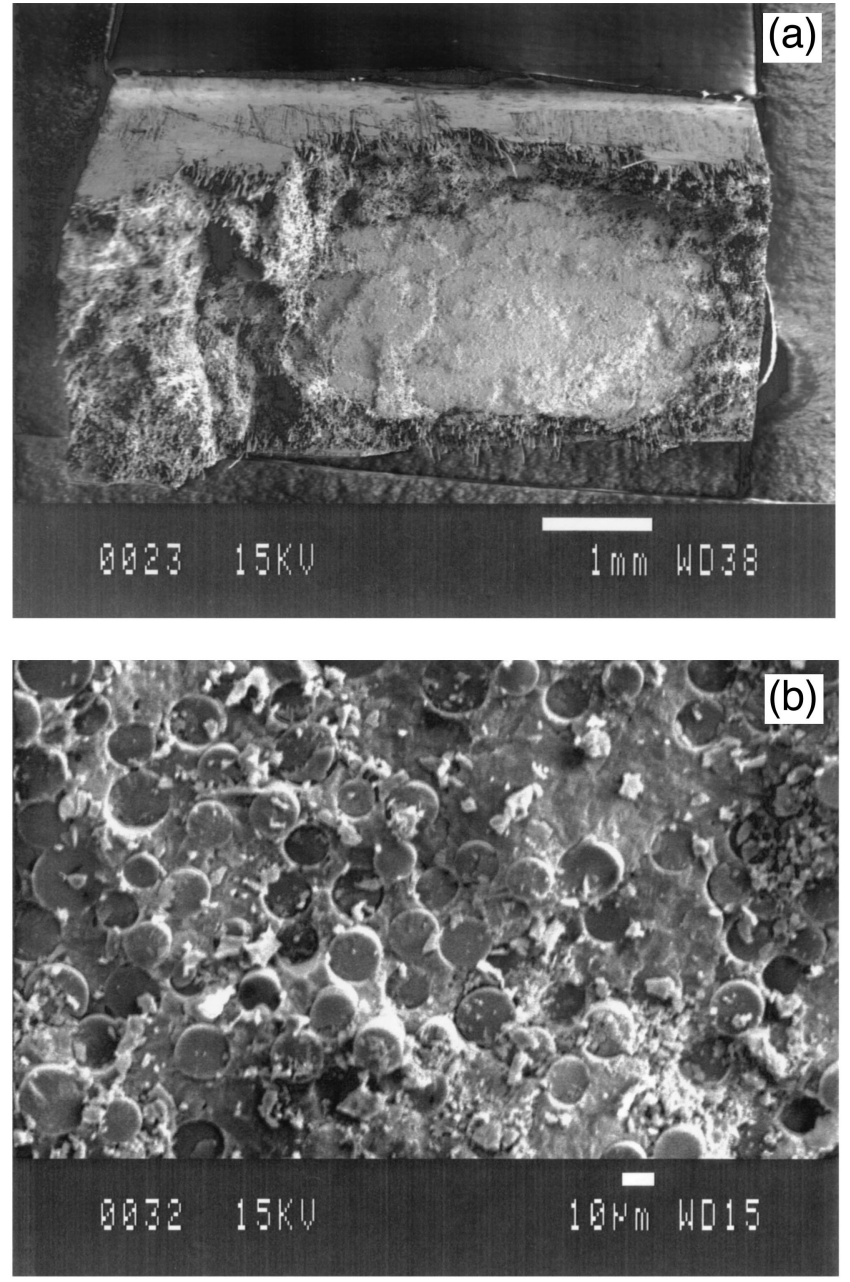

Fig. 8. Micrographs showing (a) fracture surface of specimen that was cycled to failure, and (b) close-up of centrally located zone with no fiber pull-out.

surface. This suggests that the embrittled zone formed later than the matrix cracks.

\section{(4) Comments Regarding Endurance Life}

The tension-tension fatigue behavior of the $\mathrm{SiC}_{\mathrm{f}} / \mathrm{CAS}$ II system under investigation in this paper has also been studied by a number of other investigators. Butkus et al. ${ }^{9}$ observed run-out at $10^{6}$ cycles for fatigue stresses up $200 \mathrm{MPa}$ at a loading frequency of $10 \mathrm{~Hz}$. Holmes and $\mathrm{Cho}^{19}$ found that fatigue failure of a specimen cycled at $25 \mathrm{~Hz}$ between stress levels of 10 and $180 \mathrm{MPa}$ occurred at $3.21 \times 10^{6}$ cycles. Karandikar and $\mathrm{Chou}^{17}$ conducted fatigue experiments up to

Table I. Average Matrix Crack Spacing for Specimens Fatigued up to Different Maximum Stress Values ${ }^{\dagger}$

\begin{tabular}{llc}
\hline$\sigma_{\max }(\mathrm{MPa})$ & \multicolumn{1}{c}{$s(\mu \mathrm{m})$} & Number of cycles \\
\hline $160^{\ddagger}$ & $1710^{\S}$ & $10^{8}$ \\
$200^{\ddagger}$ & $722^{\S}$ & $10^{8}$ \\
212 & 175 & $10^{8}$ \\
215 & 655 & $7.7 \times 10^{5}$ \\
220 & $245^{\ddagger}, 248,508$ & $4.3^{\ddagger} \times 10^{5}, 7.6 \times 10^{5}, 5.0 \times 10^{6}$ \\
240 & 201 & $3.0 \times 10^{5}$ \\
260 & 361 & $1.5 \times 10^{5}$ \\
280 & 308 & $6.9 \times 10^{4}$ \\
\hline
\end{tabular}

If more than one value is given, several specimens were tested for that condition. *Experiments conducted at $500 \mathrm{~Hz}$. ${ }^{8}$ Cracks mainly restricted to matrix-rich regions.
$10^{6}$ cycles $(10 \mathrm{~Hz})$. Although a significant number of matrix cracks developed and the modulus of the composite decreased significantly, no fatigue failures occurred within $10^{6}$ cycles (the maximum stress examined was $195 \mathrm{MPa}$ ). Similar results were obtained by Pryce and Smith: ${ }^{16}$ run-out at $10^{5}$ cycles for a maximum stress of $200 \mathrm{MPa}$ and a loading frequency of $10 \mathrm{~Hz}$. Rousseau ${ }^{15}$ observed that $\varepsilon^{*}$ increased during cycling, and suggested that $\varepsilon^{*}$ might be a useful indicator of fatigue damage. Rousseau et $a l .{ }^{40}$ conducted fatigue experiments inside a scanning electron microscope at $\sim 1 \mathrm{~Hz}$. They observed that the average fiber pull-out length decreased as the maximum stress level was decreased, i.e., with longer fatigue life. Holmes et $a l .{ }^{11}$ found that the fatigue limit (defined as run-out at $5 \times 10^{6}$ cycles) decreased from $240 \mathrm{MPa}$ at $10 \mathrm{~Hz}$ to $180 \mathrm{MPa}$ at 350 $\mathrm{Hz}$. In other words, an increase in loading frequency reduced fatigue life. Evans et al. ${ }^{4}$ measured a fatigue limit of $325 \mathrm{MPa}$, when run-out was defined as low as $2 \times 10^{4}$ cycles. Interestingly, their low cycle fatigue limit correlated well with predictions from the interfacial shear stress based model of Rouby and Reynaud. $^{27}$

From the experimental studies described above, it is obvious that it is necessary to have some relevant criteria to determine whether a measured run-out fatigue limit is likely to be a true endurance fatigue limit. We suggest that the term 'fatigue limit' should only be used if both of the following criteria are fulfilled:

(1) The number of cycles defined as run-out should exceed the highest number of cycles that cause fatigue failure by at least a factor of ten.

(2) All damage indicators, i.e., the material response related to the state of damage (such as stiffness and frictional heating), should remain unchanged during the last $90 \%$ of the number of cycles defined as run-out.

The former criterion ensures that the number of cycles defined as run-out is sufficiently high so that the fatigue limit can readily be identified. The latter criterion is to ensure that the evolution of damage has saturated so that further fatigue damage is unlikely and, consequently, that the measured fatigue limit is a true fatigue limit.

The results from our study fulfill criterion 1 , but not criterion 2 . Thus, we conclude that, although a plateau exists on the $S-N$ curve, it is unlikely to be a true endurance fatigue limit. Fatigue damage is still evolving at $10^{8}$ cycles. Several degradation mechanisms can exist on very different scales of fatigue cycles, leading to steps in the $S-N$ curve. For CMCs with weakly bonded interfaces, it is expected that a true fatigue limit can only exist if matrix cracking is absent or limited to matrix-rich regions so that interfacial debonding and sliding does not occur.

\section{Conclusions}

(1) For the unidirectional $\mathrm{SiC}_{\mathrm{f}} / \mathrm{CAS}$ II composite examined, a clear run-out fatigue limit $\left(10^{8}\right.$ cycles $)$ was found at $212 \mathrm{MPa}$ for experiments conducted at a loading frequency of $200 \mathrm{~Hz}$.

(2) Matrix cracking was a necessary, but not sufficient, damage state to cause fatigue failure.

(3) At $10^{8}$ cycles, microstructural damage was still evolving, as evidenced by changes in specimen temperature (internal heating) and cyclic stress-strain modulus. It is, therefore, unlikely that the $10^{8}$ fatigue limit is a true endurance fatigue limit.

(4) The fracture surfaces of all specimens that failed during high-frequency fatigue showed a central core with no fiber pull-out, suggesting that the composite loses its ability for stress redistribution within the interior of the specimens.

\section{Appendix}

In this appendix, a simple estimate is made of the maximum dynamic stress that the tensile test specimens experience due to high loading frequencies. Consider a tensile specimen of length, 
$L$; width, $B$; and thickness, $H$. One of the specimen ends is held rigidly in a grip that does not move. The other end of the specimen is firmly attached to another grip traveling in a sinusoidal wave form with the loading frequency, $f$. The displacement, $\delta$, and thus the maximum dynamic stress are largest at the moving grip

$$
\delta(t)=\frac{\delta_{\max }}{2}-\delta_{\max } \cos (2 \pi f t)
$$

where $t$ is the time and $\delta_{\max }$ the maximum displacement of the bar (at the moving grip), which can be related to the maximum strain $\varepsilon_{\max }$ as

$$
\delta_{\max }=\varepsilon_{\max } L
$$

The acceleration, $a(t)$, at the moving grip is

$$
a(t)=\frac{d^{2} \delta(t)}{d t^{2}}=4 \pi^{2} f^{2} \delta_{\max } \cos (2 \pi f t)
$$

Thus, the maximum acceleration, $a_{\max }$, is

$$
a_{\max }=4 \pi^{2} f^{2} \delta_{\max }
$$

An upper bound for the dynamic stress $\sigma_{\max }^{\text {dyn }}$ is obtained by assuming that the entire mass of the bar, $m$, is subjected to the maximum acceleration. Then the first law of Newton gives

$$
\sigma_{\max }^{\mathrm{dyn}} B H=m a_{\max }=L B H \rho a_{\max }
$$

where $\rho$ is the density of the bar. Combining (A-2), (A-4), and (A-5) gives the maximum dynamic stress as

$$
\sigma_{\max }^{\text {dyn }}=4 \pi^{2} f^{2} \rho \varepsilon_{\max } L^{2}
$$

Inserting the worst case parameters $\left(f=500 \mathrm{~s}^{-1}, \rho \approx 2.5 \times 10^{3}\right.$ $\mathrm{kg} \cdot \mathrm{m}^{-3}, \varepsilon_{\max } \approx 0.3 \times 10^{-2}$, and $L \approx 0.1 \mathrm{~m}$ ) gives $\sigma_{\max }^{\mathrm{dyn}}=0.7$ $\mathrm{MPa}$. Using (as in most of the experiments) $f=200 \mathrm{~s}^{-1}$ instead gives $\sigma_{\max }^{\text {dyn }}=0.1 \mathrm{MPa}$.

\section{Acknowledgments}

Useful discussions with Dr. Xin Wu, and Professors Jim Barber and Ramesh Talreja are acknowledged.

\section{References}

${ }^{1}$ C. M. Cady, T. J. Mackin, and A. G. Evans, "Silicon Carbide/Calcium Aluminosilicate: A Notch-Insensitive Ceramic Matrix Composite," J. Am. Ceram. Soc., 78 77-88 (1995).

${ }^{2}$ J. W. Holmes and X. Wu, "Elevated Temperature Creep Behavior of Continuous Fiber-Reinforced Ceramics"; pp. 193-260 in Elevated Temperature Behavior of Ceramic Matrix Composites. Edited by S. V. Nair and K. Jakus. Butterworth, Boston, MA, 1995

${ }^{3}$ J. W. Holmes and B. F. Sørensen, "Fatigue Behavior of Continuous FiberReinforced Ceramic Matrix Composites"; see Ref. 2, pp. 261-326.

${ }^{4}$ A. G. Evans, F. W. Zok, and R. M. McMeeking, "Fatigue of Ceramic Matrix Composites," Acta Metall. Mater., 43, 859-75 (1995).

${ }^{5}$ A. G. Evans, "Design and Life Prediction Issues for High-Temperature Engineering Ceramics and Their Composites," Acta Mater., 45, 23-40 (1997).

${ }^{6}$ K. M. Prewo, "Fatigue and Stress Rupture of Silicon Carbide Fiber-Reinforced Glass-Ceramics," J. Mater. Sci., 22, 2695-701 (1987).

${ }^{7}$ L. P. Zawada, L. M. Butkus, and G. A. Hartman, "Room-Temperature Tensile and Fatigue Properties of Silicon Carbide Fiber-Reinforced Aluminosilicate Glass," Ceram. Eng. Sci. Proc., 11, 1592-606 (1990).

${ }^{8}$ T. Kotil, J. W. Holmes, and M. Comninou, "Origin of Hysteresis Observed during Fatigue of Ceramic Matrix Composites," J. Am. Ceram. Soc., 73, 1879-83 (1990).

${ }^{9}$ L. M. Butkus, L. P. Zawada, and G. A. Hartman, "Room-Temperature Tensile and Fatigue Properties of Silicon-Carbide Fiber-Reinforced Ceramic Matrix Composites", Aeromat', 90, Advanced Aerospace Materials/Processes Conference (Long Beach, CA, May 1990). 1990.

${ }^{10}$ B. F. Sørensen and J. W. Holmes, "Effect of Loading Rate on the Monotonic Tensile Behavior and Matrix Cracking of a Fiber-Reinforced Ceramic," J. Am Ceram. Soc., 79, 313-20 (1996).
${ }^{11}$ J. W. Holmes, X. Wu, and B. F. Sørensen, "Frequency Dependency of Fatigue Life and Internal Heating of a Fiber-Reinforced Ceramic Matrix Composite," J. Am. Ceram. Soc., 77, 3284-86 (1994).

${ }^{12}$ W. R. Moschelle, "Load Ratio Effects on the Fatigue Behavior of Silicon Carbide Fiber-Reinforced Silicon Carbide Composites," Ceram. Eng. Sci. Proc., 15, 13-22 (1994).

${ }^{13}$ B. F. Sørensen and J. W. Holmes, "Influence of Stress Ratio on the Fatigue Life of a Continuous Fiber-Reinforced Ceramic Matrix Composite," manuscript in preparation.

${ }^{14}$ S. F. Shuler, J. W. Holmes, X. Wu, and D. Roach, "Influence of Loading Frequency on the Room-Temperature Fatigue of a Carbon-Fiber/SiC-Matrix Composite," J. Am. Ceram. Soc., 76, 2327-36 (1993).

${ }^{15} \mathrm{C}$. Q. Rousseau, "Monotonic and Cyclic Behavior of a Silicon Carbide/CalciumAluminosilicate Ceramic Composite"; pp. 136-51 in Thermal and Mechanical Behavior of Metal Matrix and Ceramic Matrix Composites, ASTM STP 1008. Edited by J. M. Kennedy, H. H. Moeller, and W. S. Johnson. ASTM, Philadelphia, PA, 1990.

${ }^{16}$ A. W. Pryce and P. A. Smith, "Matrix Cracking in Unidirectional Ceramic Matrix Composites under Quasi-Static and Cyclic Loading," Acta Metall. Mater., 41, 1269-81 (1993).

${ }^{17}$ P. Karandikar and T.-W. Chou, "Damage Development and Moduli Reductions in Nicalon-Calcium Aluminosilicate Composites under Static Fatigue and Cyclic Fatigue," J. Am. Ceram. Soc., 76, 1720-28 (1993).

${ }^{18} \mathrm{~J}$. W. Holmes and S. F. Shuler, "Temperature Rise During Fatigue of FiberReinforced Ceramics," J. Mater. Sci. Lett., 9, 1290-91 (1990).

${ }^{19}$ J. W. Holmes and C. Cho, "Experimental Observations of Frictional Heating in Fiber-Reinforced Ceramics," J. Am. Ceram. Soc., 75, 929-38 (1992).

${ }^{20} \mathrm{~W}$. P. Keith and K. T. Kedward, "The Stress-Strain Behavior of a Porous Unidirectional Ceramic Matrix Composite," Composites, 26, 163-74 (1995).

${ }^{21}$ C. Cho, J. W. Holmes, and J. R. Barber, "Estimation of Interfacial Shear in Ceramic Composites from Frictional Heating Measurements," J. Am. Ceram. Soc., 74, 2808-18 (1991)

${ }^{22}$ P. D. Jero and R. J. Kerans, "The Contribution of Interfacial Roughness to Sliding Friction of Ceramic Fibers in a Glass Matrix," Scr. Metall. Mater., 24, 2315-18 (1990).

${ }^{23}$ S. Q. Guo and Y. Kagawa, "Characterization of Interface Sliding Damage in SiC Fiber-Reinforced Ti-15-3 Matrix Composite by Cyclic Fatigue," Acta Mater., 45, 2257-70 (1997).

${ }^{24}$ M. Sutcu, "Statistical Fibre Failure and Single Crack Behavior in Uniaxially Reinforced Ceramic Composites," J. Mater. Sci., 23, 928-33 (1988).

${ }^{25}$ M. D. Thouless and A. G. Evans, "Effects of Pull-Out on the Mechanical Properties of Ceramic Matrix Composites," Acta Metall., 36, 517-22 (1988).

${ }^{26}$ W. A. Curtin, "Theory of Mechanical Properties of Ceramic Matrix Composites," J. Am. Ceram. Soc., 74, 2837-45 (1991).

${ }^{27}$ D. Rouby and P. Reynaud, "Fatigue Behavior Related to the Interface Modification during Load Cycling in Ceramic Matrix Composites," Comp. Sci. Technol., 48, 109-18 (1993)

${ }^{28}$ W. L. Morris, B. N. Cox, D. B. Marshall, R. V. Inman, and M. R. James, "Fatigue Mechanisms in Graphite/SiC Composites at Room and High Temperature," J. Am. Ceram. Soc., 77, 794-800 (1994).

${ }^{29}$ N. Chawla, Y. K. Tur, J. W. Holmes, and J. R. Barbar, "High-Frequency Fatigue Behavior of Woven-Fiber-Fabric-Reinforced Polymer-Derived Ceramic Matrix Composite," J. Am. Ceram. Soc., 81, 1221-30 (1998).

${ }^{30}$ S. F. Shuler, J. W. Holmes, X. Wu, and D. Roach, "Influence of Loading Frequency on the Room-Temperature Fatigue of a Carbon-Fiber/SiC-Matrix Composite," J. Am. Ceram. Soc., 76, 2327-36 (1993).

${ }^{31}$ A. G. Evans, F. W. Zok, R. M. McMeeking, and Z. Du, "Models of HighTemperature, Environmentally Assisted Embrittlement in Ceramic Matrix Composites," J. Am. Ceram. Soc., 79, 2345-55 (1996).

${ }^{32}$ S. M. Spearing, F. W. Zok, and A. G. Evans, "Stress Corrosion Cracking in a Unidirectional Ceramic Matrix Composite," J. Am. Ceram. Soc., 77, 562-70 (1994).

${ }^{33}$ R. W. Goettler and K. T. Faber, "Interfacial Shear Stress in Fiber-Reinforced Glasses," Compos. Sci. Technol., 37, 129-47 (1989).

${ }^{34} \mathrm{~J}$. W. Holmes, "A Technique for Tensile Fatigue and Creep Testing of Fiber-Reinforced Ceramics," J. Compos. Mater., 26, 915-23 (1992).

${ }^{35}$ B. F. Sørensen and R. Talreja, "Analysis of Damage in Ceramic Matrix Composites," Int. J. Damage Mech., 2, 246-71 (1993).

${ }^{36}$ D. S. Beyerle, S. M. Spearing, F. W. Zok, and A. G. Evans, "Damage and Failure in Unidirectional Ceramic Matrix Composites," J. Am. Ceram. Soc., 75, 2719-25 (1992).

${ }^{37}$ B. F. Sørensen and J. W. Holmes, "Fatigue of Continuous Fiber-Reinforced Ceramic Matrix Composites: Review of Mechanisms and Models"; pp. 487-500 in Proceedings of the International Symposium on Fatigue under Thermal and Mechanical Loading, Edited by J. Bressers and L. Remy. Kluwer, Dordrecht, The Netherlands, 1996.

${ }^{38}$ D. Koch and G. Grathwohl, "S-Curve-Behavior and Temperature Increase of Ceramic Matrix Composites during Fatigue Testing"; pp. 419-24 in HighTemperature Ceramic Matrix Composites I: Design, Durability and Performance. Edited by A. G. Evans and R. Naslain. American Ceramic Society, Westerville, OH, 1995.

${ }^{39}$ B. F. Sørensen, J. W. Holmes, and E. L. Vanswijgenhoven, "Rate of Strength Decrease of Fiber-Reinforced Ceramic Matrix Composites During Fatigue," J. Am. Ceram. Soc., 83, 1469-75 (2000).

${ }^{40}$ C. Q. Rousseau, D. L. Davidson, and J. B. Campbell, "The Micromechanics of Ambient Temperature Cyclic Loading Fatigue in a Composite of CAS Glass Ceramic Reinforced with Nicalon Fibers," J. Compos. Technol. Res., 16, 115-26 (1996). 